\title{
REVIEW ON CELECOXIB: A ORAL
}

\section{COX-2 INHIBITOR}

\author{
Priya G. Shete ${ }^{1 *}$, Neha G. Shete ${ }^{2}$, Dattaprasad N. Kumbhakaran ${ }^{2}$, \\ Namrata S. Mane ${ }^{1}$, Vishwa S. Padole ${ }^{1}$ and Ravi P. Kalsait ${ }^{1}$ \\ ${ }^{1}$ Nagpur College of Pharmacy, Wanadongri, Hingna Road, \\ Nagpur-441 110, Maharashtra, Mumbai, India. \\ ${ }^{2}$ Department of Pharmaceutics, Agnihotri College of Pharmacy, \\ Wardha-442 301 Maharashtra, Mumbai, India.
}

\begin{abstract}
Nonsteroidal anti-inflammatory drugs (NSAIDs) have been widely used for the treatment of arthritic conditions. Drugs in this heterogeneous class alleviate pain and inflammation by inhibiting cyclooxygenase-2 (COX-2). Cyclooxygenase-1 (COX-1) inhibition has traditionally been associated with increased gastrointestinal (GI) harm, whereas increased COX-2 selectivity has more recently become associated with greater risk of cardiovascular (CV) harm. When the entirety of data is considered, NSAIDs can be seen to exhibit a range of COX isoform selectivity, with all oral NSAIDs appearing to be associated with an increase in (CV) cardiovascular events. Non-steroidal anti-inflammatory drugs presently are the most widely used drugs in medicine and are the most frequent cause of adverse drug reactions, affecting multiple systems, mainly gastric, renal \& cardiovascular, which is a major public health concern. Previous studies report, that selective COX-2 inhibitors are safer when compared to non-selective cyclooxygenase inhibitors. But, recent studies reveal, that the safety of these selective COX-2 inhibitors is not much better than that of conventional NSAIDs. In view of the wider usage of selective COX-2 inhibitor, celecoxib in day to day practice by many clinicians, the study has been taken up to report, whether selective COX-2 inhibitor celecoxib has got any advantages over conventional NSAIDs or not.It is approved in one or more countries worldwide for the relief of the signs and symptoms of osteoarthritis (OA), rheumatoid arthritis (RA), juvenile rheumatoid arthritis (in patients aged $>$ or $=2$ years) and ankylosing spondylitis (AS), the management of acute pain in adults, the treatment of primary dysmenorrhoea and the reduction in the number of adenomatous colorectal polyps in familial adenomatous polyposis.
\end{abstract}

Keywords: Celecoxib, nonsteroidal anti-inflammatory drug, NSAIDs and ADR.

\section{INTRODUCTION}

Nonsteroidal anti-inflammatory drugs (NSAIDs) have been widely prescribed for decades to reduce pain and inflammation in patients with chronic arthritic conditions. NSAIDs provide symptomatic relief by inhibiting cyclooxygenase (COX) enzymes, with a subsequent reduction in the prostaglandin mediators of pain. Beneficial effects were believed to be predominantly mediated through inhibition of the largely inducible form of the enzyme, known as cyclooxygenase-2 (COX-2), whereas housekeeping functions, such as gastric homeostasis, were believed to be largely mediated through cyclooxygenase-1 (COX1) 1,2 .

While all NSAIDs must inhibit COX-2 to have any effect on pain/inflammatory processes, NSAIDs are a chemically diverse group of 
medicines with significant heterogeneity in chemical structure and properties ${ }^{3,4}$. They show substantial variation in selectivity for COX-2 over COX-1, with this variable being best considered as continuous rather than simply dichotomous, where medicines are presented as either COX-2 selective or nonselective ${ }^{5}$

Medicines with proprietary names ending in "coxib", such as celecoxib or etoricoxib, are traditionally thought of as the most selective in their effect on the COX-2 enzymes and were developed to reduce gastrointestinal (GI) toxicity associated with the use of traditional NSAIDs, but they have been characterized by some as having less favorable cardiovascular (CV) profiles than agents that are nonselective ${ }^{5}$. The term coxib, however, has no pharmacological meaning, and different in vitro assay systems, measuring selectivity ratios for COX-1 and COX-2 enzymes, can produce markedly different results ${ }^{6,7}$.

Celecoxib capsules (100 and $200 \mathrm{mg}$ ) have been fully subsidised in New Zealand, without restriction, since June 1,2017 . Celecoxib is generally considered to be equally effective as naproxen, ibuprofen and diclofenac in reducing pain and inflammation ${ }^{8,9}$

Celecoxib can be considered for the treatment of patients with ${ }^{10}$

- Acute pain

- Osteoarthritis

- Rheumatoid arthritis

- Ankylosing spondylitis

- Primary dysmenorrhoea

As with other NSAIDs, celecoxib is contraindicated in patients with NSAID hypersensitivity, e.g. those with asthma, urticaria, angioedema or rhinitis that is caused by a NSAID, including aspirin. ${ }^{10}$ Celecoxib is also contraindicated in patients with ischaemic heart disease, cerebrovascular disease, peripheral artery disease, mild to severe heart failure, active gastrointestinal ulceration or bleeding or inflammatory bowel disease. ${ }^{10}$ Caution is advised when considering the use of celecoxib in older patients and patients with reduced renal function, patients at risk of gastrointestinal bleeding and those taking medicines that may interact with NSAIDs, e.g. diuretics and ACE inhibitors. ${ }^{10}$ Celecoxib is associated with an increased risk of cardiovascular events, but this risk is similar to that of the non-selective NSAIDs .

Older patients with osteoarthritis or rheumatoid arthritis often require long-term treatment with nonsteroidal anti-inflammatory drugs (NSAIDs) for the relief of chronic pain and inflammation. ${ }^{11,12}$ With their decreased renal function and gastrointestinal (GI) physiological changes progressing with aging, those patients are more predisposed to adverse effects of prolonged exposure to the ulcerogenic analgesics. It is well documented that the GI toxicity of NSAIDs is associated with the nonselective inhibition of the two cyclooxygenase (COX) isoforms, where COX1 inhibition prevents the production of $\mathrm{Gl}$ protective prostaglandins resulting in higher risk of ulcerogenic adverse events, while COX2 inhibition exerts anti-inflammatory and analgesic effects. ${ }^{13.14}$ Traditional NSAIDs are mostly a dual inhibitor of both COX isoforms, and hence can cause GI toxicities. Celecoxib, however, by selectively inhibiting COX-2, has the potential to reduce $\mathrm{Gl}$ adverse effects, but previous studies suggested that the unbalanced inhibition of COX or prostaglandin synthesis may pose a greater risk of cardiovascular (CV) adverse outcomes. ${ }^{15,16}$

Due to the controversies over CV safety of COX-2 specific inhibitors, rofecoxib and valdecoxib were withdrawn from the market in 2004 and 2005, respectively. ${ }^{15}$ The only remaining coxib agent, celecoxib, has been made continuously available for clinical use, despite the 2005 clinical trial results that suggested increased CV risks with its use ${ }^{17}$.

\section{Pharmacokinetics}

After oral administration, celecoxib is rapidly absorbed and achieves peak serum concentration in approximately $3 \mathrm{~h}$. It is extensively metabolized in the liver, with very little drug $(<3 \%)$ being eliminated unchanged ${ }^{18}$. The major routes of excretion for celecoxib are feces and urine ${ }^{19}$. Celecoxib is metabolized primarily through methyl hydroxylation to form hydroxycelecoxib. This reaction is largely catalyzed by CYP2C9, although CYP3A4 also plays a minor $(<25 \%)$ role ${ }^{18,20,21}$. Hydroxycelecoxib is further oxidized to form carboxycelecoxib by cytosolic alcohol dehydrogenases $\mathrm{ADH} 1$ and $\mathrm{ADH} 2^{20}$, then conjugated with glucuronic acid by UDP glucuronosyltransferases to form the 1-Oglucuronide. None of the metabolites are pharmacologically active ${ }^{21}$.

As celecoxib metabolism is predominantly mediated by CYP2C9, polymorphisms in CYP2C9 are likely to have a direct impact on celecoxib pharmacokinetics and variability in drug responses. Individuals who are poor metabolizers of CYP2C9 substrates (e.g. CYP2C9*3 allele carriers) have an increased exposure to celecoxib when compared with those with normal CYP2C9 activity ${ }^{20,22}$ (see 'Pharmacogenomics' section). Drugs that inhibit CYP2C9 should therefore be used with caution in patients taking celecoxib. 
Although not a substrate of CYP2D6, celecoxib inhibits this metabolic enzyme ${ }^{23}$. Drugs that are metabolized by CYP2D6 (e.g. metoprolol ${ }^{23}$ ) should also be used with caution in patients receiving celecoxib due to a potential risk of drug interaction.

\section{Pharmacodynamics}

Celecoxib exerts its anti-inflammatory and analgesic activities through blocking the synthesis of various inflammatory prostanoids $(P G)^{21,24,25}$. The prostanoids, which include PGs and thromboxane, are the end products of fatty acid metabolism produced by tissuespecific COX enzymatic activity. These products are important physiological and pathological mediators that are involved in a wide range of biological processes including inflammation, pain, cancer, glaucoma, osteoporosis, cardiovascular diseases, and asthma ${ }^{26}$. The production of the prostanoids (PG) is dependent on the availability of arachidonic acid (AA). Following stimulation of the cell membrane by inflammatory or mitogenic signals, the first step in $P G$ synthesis is the release of $A A$ from the cellular phospholipids through the action of either secretory (SPLA2, encoded by gene PLA2G2A) or cytoplasmic (cPLA , encoded by gene PLA2G4A) phospholipases. Once AA is released, the two isoenzymes, COX-1 (encoded by PTGS1) and COX-2 (encoded by PTGS2), catalyze the production of the prostanoids ${ }^{27}$. As indicated above, this involves two sequential reactions. The initial COX reaction converts $A A$ into $P G G_{2}$. The second reaction reduces $P G G_{2}$ to $P G_{2}$. $\mathrm{PGH}_{2}$ is then converted into active metabolites $\mathrm{PGE}_{2}$, prostacyclin $\left(\mathrm{PGI}_{2}\right)$, thromboxane $\left(T \times A_{2}\right), P G D_{2}$, and $P G F_{2 \alpha}$ by the action of tissue-specific $P G$ synthases ${ }^{28,29}$. These active metabolites interact with specific prostanoid Gprotein-coupled receptors to mediate diverse physiological responses, such as inflammation, fever, blood pressure regulation, clotting, and gastrointestinal protection.

The COX-1 and COX-2 enzymes exhibit distinct expression profiles and roles in physiological processes. COX-1 is constitutively expressed in many cell types and is the major COX isoform in gastric tissue. It is responsible for the protection of the gastric mucosa, which led to the development of the 'COX-2 hypothesis' that drugs targeted against COX-2 only would have reduced upper gastrointestinal toxicity ${ }^{24}$ Although COX-2 is highly inducible by inflammatory stimuli such as cytokines, growth factors, and tumor promoters $^{30-33}$, it is also constitutively expressed in some tissues, such as the vessel wall, the kidney, or the heart. Indeed, the depression of the physiological formation of COX-2-dependent prostanoids in these tissues has been identified as the molecular mechanism underlying the thrombotic cardiovascular complications of COX-2 inhibition $^{34}$. Seven placebocontrolled, randomized trials with three chemically distinct pdCOX-2 inhibitors, including celecoxib, have documented the cardiovascular risk. Of note, celecoxib is now used at lower doses than in the trials that showed its cardiovascular hazard. Celecoxib has 30-fold greater inhibitory activity against COX-2 compared with COX-1, and inhibits COX-1 only minimally at therapeutic concentrations ${ }^{35,36}$. Although the selectivity for COX-2 measured in vitro is lower for celecoxib compared with other drugs in the coxib class (e.g. rofecoxib, valdecoxib, lumiracoxib, and etoricoxib), it is very similar at therapeutic concentrations in vivo. Celecoxib also retains more ability to inhibit COX-1 compared with other coxibs; however, the consequences of this with regard to its therapeutic efficacy and toxicity are not well understood $^{37-39}$

\section{The Role of Celecoxib in Treatment of Psychiatric Disorders \\ Schizophrenia}

Several clinical trials have investigated the effectiveness of celecoxib in the treatment procedure of Schizophrenia ${ }^{40-45}$. In Ref $f^{40}$, the authors studied the effect of celecoxib (400 $\mathrm{mg})$ plus amisulpride (200-1000 mg) in comparison with amisulpride (200- $1000 \mathrm{mg}$ ) plus placebo. The study was performed on 49 schizophrenic patients who were diagnosed within two years of the study date, for duration of six weeks. The results show a significant difference Citation: Shalbafan M, Malekpour F, Donboli S, Shirazi E, Moridian M. The Role of Celecoxib in Treatment of Psychiatric Disorders: A Review Article. J Neurol Psychol. 2018; 6(1): 4. J NeurolPsychol 6(1): 4 (2018) Page - 02 ISSN: 2332-3469 between celecoxib group and placebo group in Global Clinical Impression Scale (CGI) and Negative, Global and Total subscales of Positive and Negative Symptom Scale (PANSS) ${ }^{40}$. In Ref ${ }^{41}$, the authors compared 60 schizophrenic patients who were randomized in risperidone 6 $\mathrm{mg} /$ day plus celecoxib $400 \mathrm{mg} /$ day and risperidone $6 \mathrm{mg} /$ day plus placebo arms for 8 weeks. It was found that celecoxib arm had superiority in Positive, General and Total scores of PANSS ${ }^{41}$. Similarly, Muller and his colleagues conducted a research on 50 schizophrenic patients who were randomized to risperidone plus celecoxib and risperidone plus placebo group for 5-weeks after washout period. They showed that patients who were in 
celecoxib group had better outcome in comparison to placebo group in the PANSS total score ${ }^{42}$. In addition a research evaluated cytokine levels in 28 schizophrenic patients who are stable on olanzapine or risperidone after 8 weeks treatment with celecoxib or placebo as adjuvant therapy. This article reported that none of the measured cytokines altered after 8 weeks ${ }^{43}$. There was another study that compared 38 symptomatic outpatient schizophrenic patients who were on treatment with atypical antipsychotics in 2 arms, celecoxib and placebo, as adjunctive therapy. Researchers reported there was no significant difference between two arms after 8 weeks in clinical symptoms or measures of disability $^{44}$. Finally, a Meta-analyzes was published recently and compared 326 schizophrenic patients, 316 patients in celecoxib groups and 310 subjects in placebo groups. The authors concluded that celecoxib could be an effective and safe choice as adjunctive therapy for psychotic symptoms of schizophrenia, particularly in first episode of illness [45]. Depression Similar to Schizophrenia, Depression has been widely studied to evaluate the effects adding celecoxib to the treatment procedure. In a sixweek study ${ }^{46}, 40$ patients who have been diagnosed as Major Depressive Disorder (MDD) were randomized into two arms, celecoxib (200 mg twice daily) or placebo plus sertraline (200 mg/day). Researchers showed that celecoxib group had better improvement in Hamilton Depression Rating Scale (HDRS) items and further reduction of Inerlukine-6 (IL$6)^{46}$. In Ref ${ }^{47, t}$ he authors compared two groups of 15 depressed female drug naive patients who were treated with celecoxib (100 mg/twice per day) or placebo in addition with sertraline for eight weeks. The authors reported significant improvement in depression symptoms through celecoxib group in comparison with placebo group in fourth week, whereas difference between two arms was not significant in eighth week ${ }^{47}$. Similarly, a sixweek study conducted to compare the effects of celecoxib ( $200 \mathrm{mg} / \mathrm{two}$ times per day) or placebo in added to fluoxetine 20 to $40 \mathrm{mg}$ per day in 40 adult patients of MDD. Researchers showed greater improvement in depressive symptoms in celecoxib arm with no difference in side effects ${ }^{48}$. Another study also evaluated the effectiveness of celecoxib $400 \mathrm{mg} / \mathrm{d}$ against placebo plus reboxetine for six weeks in 40 depressed patients who began treating after a wash-out period. It has been reported that celecoxib plus reboxetine had greater improvement in comparison to reboxetine alone $^{49}$. Finally, a Meta-analyses included 6262 subjects and its authors showed that anti-inflammatory agents, particularly celecoxib decrease depressive symptoms without increasing the risk of side effects ${ }^{50}$.

\section{Depression}

In particular populations Several researches were conducted to evaluate the effectiveness of celecoxib in subjects other than adults medically healthy depressed patients. Interestingly, two of these studies targeted depressed patients with a previously diagnosed cancer. Alamdarsaravi et al. investigated the effect of celecoxib $(400 \mathrm{mg} / \mathrm{d})$ in comparison to placebo in 40 patients with colorectal cancer and mild to moderate depression for six weeks. The authors reported a better improvement in celecoxib group $^{51}$. Similarly, Mohammadinejad and his colleagues compared depressive symptoms between two arms who were treated with celecoxib $(400 \mathrm{mg} / \mathrm{d})$ or diclofenac $(50 \mathrm{mg} / \mathrm{d})$ in 52 outpatients with breast cancer and mild to moderate depression for 6 weeks. They also, showed that celecoxib group had better improvement in depressive symptoms, significantly $^{52 .}$ In addition, another research conducted to evaluate celecoxib's effect in comparison with placebo added to an antibiotic in patients with brucellosis on depressive symptoms. Researchers showed after eight week study on 40 subjects that celecoxib had superiority to placebo ${ }^{53}$.

\section{Celecoxib Is An Effective Treatment For Patients With Ankylosing Spondylitis}

The European League Against Rheumatism (EULAR) guidelines ${ }^{54}$ advise that treatment should be tailored according to the current manifestations of the disease, including status and severity of symptoms. Disease monitoring (on an individual basis) should include patient history, clinical parameters, laboratory tests, and imaging, while non-pharmacological treatment should be based on patient education and regular exercise ${ }^{54}$. Ongoing treatment with NSAIDs, including COX-2 selective inhibitors, is recommended as firstline drug treatment with other pharmacological treatments (including analgesics, directed corticosteroid injections, and anti-tumor necrosis factor [TNF] therapy) considered if NSAIDs are ineffective ${ }^{5}$. Surgery may also be considered in more severe, non-responding patient $^{54}$.

The American College of Rheumatology (ACR) guidelines ${ }^{55}$ are similar, also strongly recommending ongoing treatment with NSAIDs and physical therapy. In the ACR guidelines, anti- TNF therapy is strongly recommended in patients who do not respond to NSAIDs as is surgery for more severe 
patients, while the guidelines strongly recommended against the use of systemic glucocorticoids ${ }^{55}$.

\section{CONCLUSION}

Celecoxib is reported to be an effective treatment for various psychiatric disorders including depression, schizophrenia. More studies with larger sample sizes are required to evaluate the use of this safe and interesting drug in psychiatric conditions and obtain an acceptable clinical suggestion. Celecoxib is one of the most well studied NSAID treatments for patients with ankylosing spondylitis with relatively strong evidence for its safety and efficacy. Recent evidence suggests that it may also have disease modifying properties. Patients with a high risk of radiographic progression may benefit from the continued use of celecoxib, even after achieving the significant improvements in clinical symptoms commonly recorded in clinical trials of this class of medicines.

\section{REFERENCES}

1. Mitchell JA, Akarasereenont $P$, Thiemermann C, Flower RJ and Vane JR. Selectivity of nonsteroidal antiinflammatory drugs as inhibitors of constitutive and inducible cyclooxygenase. Proceedings of the National Acadamy of Sciences of the United States of America. 1993;90(24):11693-11697.View at: Publisher Site | Google Scholar.

2. Vane JR, Bakhle YS and Botting RM. Cyclooxygenases 1 and 2. Annual Review of Pharmacology and Toxicology. 1998;38:97-120.View at: Publisher Site | Google Scholar.

3. Schwartz JI, Dallob AL and Larson PJ. Comparative inhibitory activity of etoricoxib, celecoxib, and diclofenac on COX-2 versus COX-1 in healthy subjects. Clinical Pharmacology and Therapeutics. 2008;48(6):745-754.View at: Publisher Site | Google Scholar.

4. Hunter TS, Robison C and Gerbino PP. Emerging evidence in NSAID pharmacology: important considerations for product selection. The American Journal of Managed Care. 2015;21(7): S139-S147. View at: Google Scholar.

5. Schmidt $M$, Lamberts $M$ and Olsen AS. Cardiovascular safety of non-aspirin nonsteroidal anti-inflammatory drugs: review and position paper by the working group for Cardiovascular Pharmacotherapy of the European Society of Cardiology. European Heart Journal - Cardiovascular
Pharmacotherapy.2016;2(2):108-118. View at: Publisher Site | Google Scholar

6. Gierse JK, Koboldt CM, Walker MC, Seibert $\mathrm{K}$ and Isakson PC. Kinetic basis for selective inhibition of cyclooxygenases. Biochemical Journal. 1999;339(3):607-614.View at: Publisher Site | Google Scholar.

7. Warner TD, Giuliano F, Vojnovic I, Bukasa A, Mitchell JA and Vane JR. Nonsteroid drug selectivities for cyclooxygenase-1 rather than cyclooxygenase- 2 are associated with human gastrointestinal toxicity: a full in vitro analysis. Proceedings of the National Academy of Sciences of the United States of America. 1999;96(13):7563-7568. View at: Publisher Site | Google Scholar.

8. Gordo AC, Walker C and Armada B. Efficacy of celecoxib versus ibuprofen for the treatment of patients with osteoarthritis of the knee: A randomized double-blind, non-inferiority trial. J Int Med Res. 2017;45:5974. http://dx.doi.org/10.1177/0300060516 673707.

9. Derry $S$ and Moore RA. Single dose oral celecoxib for acute postoperative pain in adults. Cochrane Database Syst Rev. 2013. CD004233. http://dx.doi.org /10.1002/14651858.CD004233.pub4

10. New Zealand Formulary (NZF). NZF v67. $2018 . \quad$ Available from: www.nzf.org.nz (Accessed Oct, 2017).

11. Hochberg MC, Altman RD and April KT. American College of Rheumatology 2012 recommendations for the use of nonpharmacologic and pharmacologic therapies in osteoarthritis of the hand, hip, and knee. Arthritis Care Res. 2012;64(4):465-474.

12. Zhang W, Doherty $M$ and Arden $N$. EULAR evidence based recommendations for the management of hip osteoarthritis: report of a task force of the EULAR Standing Committee for International Clinical Studies Including Therapeutics (ESCISIT)s. Ann Rheum Dis. 2005;64(5):669-681.

13. Whittle BJ. COX-1 and COX-2 products in the gut: therapeutic impact of COX-2 inhibitors. Gut. 2000;47(3):320-325.

14. Wolfe MM, Lichtenstein DR and Singh G. Gastrointestinal toxicity of nonsteroidal anti-inflammatory drugs. N Engl J Med. 1999;340(24):1888-1899.

15. Zarghi A and Arfaei S. Selective COX-2 Inhibitors: A Review of Their Structure- 
Activity Relationships. Iran J Pharm Res. 2011;10(4):655-683.

16. Fitzgerald GA. Coxibs and cardiovascular disease. N Engl J Med. 2004;351(17):1709-1711.

17. Solomon SD, Mcmurray JJ and Pfeffer MA. Cardiovascular risk associated with celecoxib in a clinical trial for colorectal adenoma prevention. N Engl J Med. 2005;352(11):1071-1080.

18. Paulson SK, Hribar JD, Liu NW, Hajdu E, Bible $\mathrm{RH}$, Jr, Piergies $\mathrm{A}$ and Karim A. Metabolism and excretion of [(14)C]celecoxib in healthy male volunteers. Drug Metab Dispos. 2000;28:308-314. [PubMed] [Google Scholar].

19. Solomon SD, Wittes J, Finn PV, Fowler $R$, Viner $J$ and Bertagnolli MM. Cardiovascular risk of celecoxib in 6 randomized placebo controlled trials: the cross trial safety analysis. Circulation. 2008;117:21042113. [PMC free article] [PubMed] [Google Scholar].

20. Sandberg M, Yasar U, Stromberg $P$, Hoog JO and Eliasson E. Oxidation of celecoxib by polymorphic cytochrome P450 2C9 and alcohol dehydrogenase. $\mathrm{Br} \mathrm{J}$ Clin Pharmacol. 2002;54:423-429. [PMC free article] [PubMed] [Google Scholar].

21. Davies NM, McLachlan AJ, Day RO and Williams KM. Clinical pharmacokinetics and pharmacodynamics of celecoxib. A selective cyclooxygenase-2 inhibitor. Clin Pharmacokinet. 2000;38:225-242. [PubMed] [Google Scholar].

22. Tang C, Shou M, Rushmore $T H$, Mei Q, Sandhu $P$ and Woolf EJ. In-vitro metabolism of celecoxib. A cyclooxygenase-2 inhibitor, by allelic variant forms of human liver microsomal cytochrome P450 2C9: correlation with CYP2C9 genotype and in-vivo pharmacokinetics. Pharmacogenetics. 20 01;11:223-235. [PubMed] [Google Scholar]

23. Werner U, Werner D, Rau T, Fromm MF, Hinz B and Brune K. Celecoxib inhibits metabolism of cytochrome P450 2D6 substrate metoprolol in humans. Clin PharmacolTher. 2003;74:130-137. [PubMed] [Google Scholar].

24. Grosser T. The pharmacology of selective inhibition of COX-2. Thromb Haemost. 2006;96:393-400. [PubMed] [Google Scholar].

25. Ricciotti E and FitzGerald GA. Prostaglandins and inflammation. Arterioscler Thromb Vasc
Biol. 2011;31:986-1000. [PMC free article] [PubMed] [Google Scholar].

26. Fitzgerald GA. Prostaglandins: modulators of inflammation and cardiovascular risk. J Clin Rheumatol. 2004;10:S12-S17. [PubMed] [Google Scholar].

27. Simmons DL, Botting RM and Hla $T$. Cyclooxygenase isozymes. The biology of prostaglandin synthesis and inhibition. Pharmacol Rev. 2004;56:387437. [PubMed] [Google Scholar]

28. Funk CD. Prostaglandins and leukotrienes: advances in eicosanoid biology. Science. 2001;294:18711875. [PubMed] [Google Scholar].

29. Murakami M, Naraba H, Tanioka T, Semmyo N, Nakatani $Y$ and Kojima F. Regulation of prostaglandin E2 biosynthesis by inducible membraneassociated prostaglandin E2 synthase that acts in concert with cyclooxygenase2. J Biol Chem. 2000;275:3278332792. [PubMed] [Google Scholar].

30. Liu J, Seibold SA, Rieke CJ, Song I, Cukier RI and Smith WL. Prostaglandin endoperoxide $\mathrm{H}$ synthases: peroxidase hydroperoxide specificity and cyclooxygenase activation. J Biol Chem. 2007;282:18233-18244.

[PubMed] [Google Scholar].

31. Smith WL and Dewitt DL. Prostaglandin endoperoxide $\mathrm{H}$ synthases-1 and 2. Advlmmunol. 1996;62:167-

215. [PubMed] [Google Scholar].

32. Smith WL and Song I. The enzymology of prostaglandin endoperoxide $\mathrm{H}$ synthases1 and -2. Prostaglandins Other Lipid Mediat. 2002;68-69:115-128.

[PubMed] [Google Scholar]

33. Thorn CF, Grosser T, Klein TE and Altman RB. PharmGKB summary: very important pharmacogene information for PTGS2. Pharmacogenet Genomics. 2011:21:607-613. [PMC free article] [PubMed] [Google Scholar].

34. Grosser T, Fries $S$ and FitzGerald GA. Biological basis for the cardiovascular consequences of COX-2 inhibition: therapeutic challenges and opportunities. J Clin Invest. 2006;116:415. [PMC free article] [PubMed] [Google Scholar].

35. Capone ML, Tacconelli S, Di Francesco L, Sacchetti A, Sciulli MG and Patrignani $P$. Pharmacodynamic of cyclooxygenase inhibitors in humans. Prostaglandins Other Lipid Mediat. 2007;82:8594. [PubMed] [Google Scholar]

36. Tacconelli S, Capone ML, Sciulli MG, Ricciotti $E$ and Patrignani $P$. The 
biochemical selectivity of novel COX-2 inhibitors in whole blood assays of COXisozyme activity. Curr Med Res Opin. 2002;18:503-

511. [PubMed] [Google Scholar].

37. Fries S, Grosser T, Price TS, Lawson JA, Kapoor $S$ and DeMarco S. Marked interindividual variability in the response to selective inhibitors of cyclooxygenase2. Gastroenterology. 2006;130:55-64. [PubMed] [Google Scholar].

38. Grosser T. Variability in the response to cyclooxygenase inhibitors: toward the individualization of nonsteroidal antiinflammatory drug therapy. J Investig Med. 2009;57:709-716.

[PubMed] [Google Scholar].

39. Rouzer CA and Marnett LJ. Cyclooxygenases: structural and functional insights. J Lipid Res. 2009;50(Suppl):S29-S34. [PMC free article] [PubMed] [Google Scholar].

40. Muller N, Krause D, Dehning S, Musil R and Schennach-Wolff R. Celecoxib treatment in an early stage of schizophrenia: results of a randomized, double-blind, placebo-controlled trial of celecoxib augmentation of amisulpride treatment. Schizophr Res. 2010;121:118124.

41. Akhondzadeh $\mathrm{S}$, Tabatabaee M, Amini $\mathrm{H}$, AhmadiAbhari SA and Abbasi $\mathrm{SH}$. Celecoxib as adjunctive therapy in schizophrenia: a double-blind, randomized and placebo-controlled trial. Schizophr Res. 2007;90:179-185.

42. Muller N, Ulmschneider M, Scheppach C, Schwarz MJ and Ackenheil M. COX-2 inhibition as a treatment approach in schizophrenia: immunological considerations and clinical effects of celecoxib add-on therapy. Eur Arch Psychiatry ClinNeurosci. 2004;254:14-22.

43. Bresee CJ, Delrahim K, Maddux RE, Dolnak $D$ and Ahmadpour $O$. The effects of celecoxib augmentation on cytokine levels in schizophrenia. Int $J$ Neuropsychopharmacol. 2006;9:343-348.

44. Rapaport MH, Delrahim KK, Bresee CJ, Maddux RE and Ahmadpour $\mathrm{O}$. Celecoxib augmentation of continuously ill patients with schizophrenia. Biol Psychiatry. 2005;57:1594-1596.

45. Zheng W, Cai DB, Yang XH, Ungvari GS and $\mathrm{Ng} \mathrm{CH}$. Adjunctive celecoxib for schizophrenia: a meta-analysis of randomized, double-blind, placebocontrolled trials. J Psychiatr Res. 2017;92: 139-146.

46. Abbasi SH, Hosseini F, Modabbernia A, Ashrafi $\mathrm{M}$ and Akhondzadeh S. Effect of celecoxib add-on treatment on symptoms and serum IL-6 concentrations in patients with major depressive disorder: randomized double-blind placebocontrolled study. J Affect Disord. 2012;141:308-314.

47. Majd M, Hashemian F, Hosseini SM, VahdatShariatpanahi $M$ and Sharifi A. A randomized, double-blind, placebocontrolled trial of celecoxib augmentation of sertraline in treatment of drug-naive depressed women: a pilot study. Iran J Pharm Res. 2015;14:891-899.

48. Akhondzadeh $S$, Jafari $S$, Raisi $F$, Nasehi AA and Ghoreishi A. Clinical trial of adjunctive celecoxib treatment in patients with major depression: a double blind and placebo controlled trial. Depress Anxiety. 2009;26:607-611.

49. Muller N, Schwarz MJ, Dehning S, Douhe $A$ and Cerovecki A. The cyclooxygenase2 inhibitor celecoxib has therapeutic effects in major depression: results of a double-blind, randomized, placebo controlled, addon pilot study to reboxetine. Mol Psychiatry. 2006;11:680684.

50. Kohler O, Benros ME, Nordentoft M, Farkouh ME and lyengar RL. Effect of anti-inflammatory treatment on depression, depressive symptoms, and adverse effects: a systematic review and meta-analysis of randomized clinical trials. JAMA Psychiatry. 2014;71:13811391.

51. Alamdarsaravi M, Ghajar A, Noorbala AA, Arbabi $M$ and Emami A. Efficacy and safety of celecoxibmonotherapy for mild to moderate depression in patients with colorectal cancer: a randomized doubleblind, placebo controlled trial. Psychiatry Res. 2017;255:59-65.

52. Mohammadinejad $P$, Arya $P$, Esfandbod $M$, Kaviani $A$ and Najafi M. Celecoxib versus diclofenac in mild to moderate depression management among breast cancer patients: a double-blind, placebocontrolled, randomized trial. Ann Pharmacother. 2015;49:953-961.

53. Jafari S, Ashrafizadeh SG, Zeinoddini A, Rasoulinejad $M$ and Entezari $P$. Celecoxib for the treatment of mild-tomoderate depression due to acute brucellosis: a double-blind, placebocontrolled, randomized trial. J Clin Pharm Ther. 2015;40:441-446.

54. Braun J, van den Berg R, Baraliakos $X$, Boehm $H$, Burgos-Vargas $R$ and Collantes-Estevez El. 2010 update of the ASAS/EULAR recommendations for the 
management of ankylosing spondylitis. Ann Rheum Dis. 2011;70(6):896-904.

55. Ward MM, Deodhar A, Akl EA, Lui A, Ermann $\mathrm{J}$ and Gensler LS. American College of Rheumatology/Spondylitis Association of America/Spondyloarthritis
Research and Treatment Network 2015 Recommendations for the Treatment of Ankylosing Spondylitis and Nonradiographic Axial Spondyloarthritis. Arthritis Rheumatol. 2016;68(2):282-298. doi: $10.1002 /$ art. 39298. 